\title{
The study of EEG Recognition of Depression on Bi-LSTM based on ERP P300
}

\author{
Yuping Zhang ${ }^{1}$, Zhigang $\mathrm{Fu}^{2}{ }^{2 *}$ \\ ${ }^{1}$ State Key Laboratory of Reliability and Intelligence of Electrical Equipment, Hebei University of Technology, Tianjin 300132, China \\ ${ }^{2}$ Physical Examination Center of the 983th Hospital of the People's Liberation Army Joint Service Support Force, Tianjin 300142, China
}

\begin{abstract}
Depression is a kind of relatively common psychological disease of among people. The extract of EEG feature is to utilize the course of development of better aided diagnosis with depression patients, so as to put forward the accurate treatment options. The traditional machine study is to directly input EEG into Neural Networks and not to consider the influence of time series for data accuracy and Bi-LSTM is not only to inherit the treatment of LSTM to timely constraint, but also combine the influence of two-way factors on neutral network, which has good computing advantage. This essay adopts a kind of the study of EEG recognition of depression on Bi-LSTM based on ERP. Compared with other model, the accuracy rate identification and classification under 16 reaches $80.6 \%$ with good credit after the improvement of the BiLSTM.
\end{abstract}

\section{Introduction}

With the increasing speed of modern rhythm, people are corresponding into the relatively faster condition in the living and working rhythm and after that the burden under the shoulder is also corresponding heavier, which leads to psychological disease with different degree for many people[1]. Among it, the most common disease is depression, which has exceeded 300 million patients around the globe. According to WHO, there are nearly one billion people who are tortured by the disease at present, and the disease will become the second largest disease next to cardiovascular disease till the middle of $21^{\text {st }}$ century. And the depression will become another important psychosomatic diseases after cardiovascular disease and tumour.

As far as the diagnosis of depression is concerned, various depression quantification tables have become the mainstream diagnostic methods. Common measurement methods is Milton quantification table[2]. The Webster Adult Intelligence Scale (WAIS-RC), the Montreal Cognitive Assessment Scale (MoCA), and the Wisconsin Card Classification Test (WCST) are commonly used in the study of cognitive function in depression. Although Hamilton depression scale can better reflect the whole recognition function and each side, its operational procedure is miscellaneous with trifles and relatively bigger impact by subjective tester and cannot objectively and accurately be confirmed for patients.

Therefore, there is an urgent need to find an objective indicator that can more accurately diagnose the development of depression patients. The development of event-related potential (ERP) provides a new direction for the diagnosis of depression. Coupled with the use of
Bi-LSTM, it can solve the trouble of manually selecting the digital features of EEG signals in traditional machine learning. And the time dynamic problem in the EEG classification, at the same time, it can take into account the forward and backward factors to make the classification results more accurate.

\section{Event-related potential theory and data collection}

\subsection{Event-related potential theory}

ERP is a potential change on the brain produced as time goes by external stimuli events or psychological events, which can be tested at the extra-cranial as well as extract and analyse through the overlay technique of wave filtering and signal, which is a kind of wide application at special brain in the field of neutral science. The p300 ERP belongs to endogenous potential, which is related to the reaction of one person to stimuli event, therefore, it can be evaluated the recognition of depression patients. P300 amplitude shows the degree of calling resources when brain processes the information of input[4], and the incubation period is related to the reaction speed of brain to stimuli[5]. Large amount of study shows that ERP has better reference value in the diagnosis and advanced in-habitation of depression. Hansenne[6] shows that P300 amplitude is universally lowered among the depression patients who have ever the suicidal sense or behaviour in depressive disorder while P300 amplitude is much more lowered among the patients who have concurrent suicide.

\footnotetext{
* Corresponding author: fu254@126.com
} 


\subsection{Data collection}

P300 belongs to endogenous component among ERP, which produces certain potential change on the brain under the related auditory or visual stimuli. This adopted stimuli method is to oddball classic flash experimental paradigms. The stimuli interface is designed to show as figure 1 .

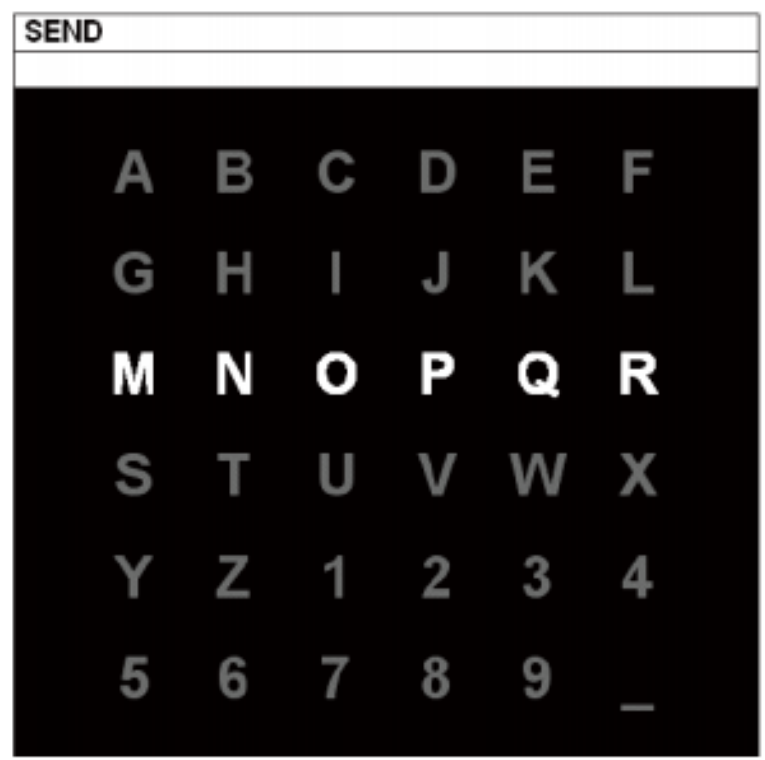

Fig.1 P300 visual evoked stimulation interface

In the experiment, the testee focuses on the one of different 36 characters. To show users the character matrix of $6 \times 6$. The task of testee is to concentrate on the character of word designated by investigator (that is input a character for once). All the rows and columns of this matrix are continuous random enforced with the frequency of $5.7 \mathrm{~Hz}$. There are 2 kinds among the 12 enforced rows and columns which include the character required (that is, a joint row and a specific column). The aroused response by these uncommon stimuli (that is, 2 of 12 stimuli the character required) is quite different from the one which is not including expected character stimuli to induce P300 potential. At the end, the data got is 80 groups of normal control and 90 groups of depression patients. Through analysing the robust ability between collection and signal, reduce from the first beginning of 32 channels to 16 channels, it separately adopts the channel signal of $\mathrm{Fz}, \mathrm{Cz}, \mathrm{Pz}, \mathrm{Oz}, \mathrm{P} 3, \mathrm{P} 4, \mathrm{P} 7$, $\mathrm{P} 8, \mathrm{FC} 1, \mathrm{FC} 2, \mathrm{C} 3, \mathrm{C} 4, \mathrm{CP} 1, \mathrm{CP} 2, \mathrm{O} 1$ and $\mathrm{O} 2$.

\section{Model construction}

The structure of a recurrent neural network includes an input layer, a recurrent layer, a fully connected layer, and an output layer. The appearance of the recurrent layer is different from the structure of other neural networks. In addition to the external RNN cycle, the LSTM cycle network also has an internal "LSTM cell" cycle (selfloop). Therefore, LSTM is not to apply a element-byelement non-linearity after the affine transformation of inputting and loop unit. Similar to common loop network, each unit has the same input and output, there are more parameters and unit system of door control to control information flow. The cell diagram of LSTM circulation network can be shown in the figure 2 .

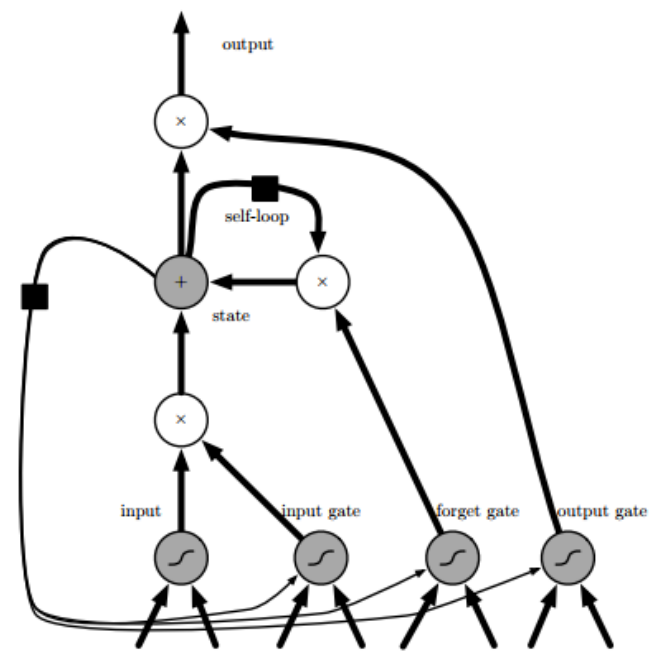

Fig.2 cell diagram of LSTM circulation network

However, the weight function of self-loop set the weight of (forget gate) $\mathrm{f}_{i}^{(t)}$ (time $\mathrm{t}$ and cell $i$ ) and sigmoid unit as the value of $0 \mathrm{tol}$ :

$$
\mathrm{f}_{i}^{(t)}=\sigma \quad\left(\mathrm{b}_{i}^{f}+\sum_{j} U_{i, j}^{f} x_{j}^{(t)}+\sum_{j} W_{i, j}^{f} h_{j}^{(t-1)}\right)
$$

Among which, $x^{(t)}$ is current input vector, $h^{t}$ is current hidden layer vector, $\mathrm{h}^{\mathrm{t}}$ includes all the output of LSTM cell. $b^{f}, U^{f}, w^{f}$ is separately offset, input weights, and forget gate loop weights. Therefore, the inner state of LSTM cell is update with following, among which there is a conditioning of self-loop weight $\mathrm{f}_{i}^{(t)}$ :

$$
\mathrm{s}_{i}^{t}=\mathrm{f}_{i}^{(t)} \mathrm{s}_{i}^{(t-1)}+\mathrm{g}_{i}^{(t)} \sigma \quad\left(\mathrm{b}_{i}+\sum_{j} U_{i, j}^{f} x_{j}^{(t)}+\sum_{j} W_{i, j}^{f} h_{j}^{(t-1)}\right)
$$

Among which, $b, U, W$ is separately offset, input weights, and forget gate loop weights. The external input gate unit $\mathrm{g}_{i}^{(t)}$ is update with the mode of similar forget gate (using sigmoid to obtain the value between 0 and 1 ), but has its own parameter:

$$
\mathrm{g}_{i}^{(t)}=\sigma \quad\left(\mathrm{b}_{i}^{g}+\sum_{j} U_{i, j}^{g} x_{j}^{(t)}+\sum_{j} W_{i, j}^{g} h_{j}^{(t-1)}\right)
$$

The output $h_{i}^{(t)}$ of LSTM cell can also be closed by output gate $q_{i}^{(t)} \quad$ (using sigmoid unit as door control):

$$
\begin{gathered}
h_{i}^{(t)}=\tanh \left(\mathrm{s}_{i}^{(t)}\right) \mathrm{q}_{i}^{(t)} \\
q_{i}^{(t)}=\sigma \quad\left(\mathrm{b}_{i}^{o}+\sum_{j} U_{i, j}^{o} x_{j}^{(t)}+\sum_{j} W_{i, j}^{o} h_{j}^{(t-1)}\right)
\end{gathered}
$$

Among which, $b^{\circ}, U_{i}^{o}, w^{\circ}$ is separately offset, input weights, and forget gate loop weights. Among these variants, you can choose to use cell state $s_{i}^{(t)}$ as its additional input and its weight, input to the third gate of unit $i$, shown as figure 2 . These requires three additional parameters. 


\section{Consequence}

\subsection{0ne-way classification of LSTM to P300 signal}

After pre-treatment of collecting 16-lead signal, every testee extracts 1800 signal feature, and choose at random 1400 feature signals as its train set and 400 feature signals as its test set in accordance with proportion of train set and test set is 4:1. Enter one by one LSTM for output of classification result. The experiment is repeated 10 times, and the average value of the classification results is selected as the final experimental classification result

The ability of the sequence signal of circulation neutral network process gets a certain application on the problems of analysing EEG. The classic one -way LSTM of accuracy result to P300 classification is shown as figure 1, when the hidden neurons are 180, and the studying speed rate is 0.001 , the classification accuracy of LSTM reaches $78.52 \%$ with the maximum.

Table 1 classification accuracy of one-way LSTM

\begin{tabular}{lll}
\hline Hidden Neurons & Learning Rate & Accuracy \\
\hline 100 & 0.003 & $72.43 \%$ \\
100 & 0.005 & $69.23 \%$ \\
150 & 0.001 & $76.78 \%$ \\
150 & 0.003 & $73.45 \%$ \\
150 & 0.005 & $68.31 \%$ \\
180 & 0.001 & $\mathbf{7 8 . 5 2 \%}$ \\
180 & 0.003 & $73.78 \%$ \\
180 & 0.005 & $68.23 \%$ \\
200 & 0.001 & $73.12 \%$ \\
200 & 0.003 & $69.61 \%$ \\
200 & 0.005 & $67.45 \%$ \\
\hline
\end{tabular}

We can see from the result, the circulation neutron network is higher than that of CNN in the classification accuracy of P300 signal, which also finds the advantage of RNN in the process of sequence signal.

\subsection{Bi-LSTM classification on P300 signal}

Place the figure as close as possible after the point where it is first referenced in the text. If there is a large number of figures and tables it might be necessary to place some before their text citation. If a figure or table is too large to fit into one column, it can be centred across both columns at the top or the bottom of the page. overlapping up and down. The output is decided by the state of RNN hidden layer, shown as figure 3.

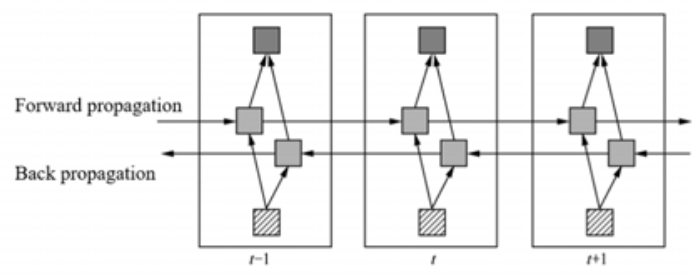

Fig.3 basic structure of Bi-LSTM

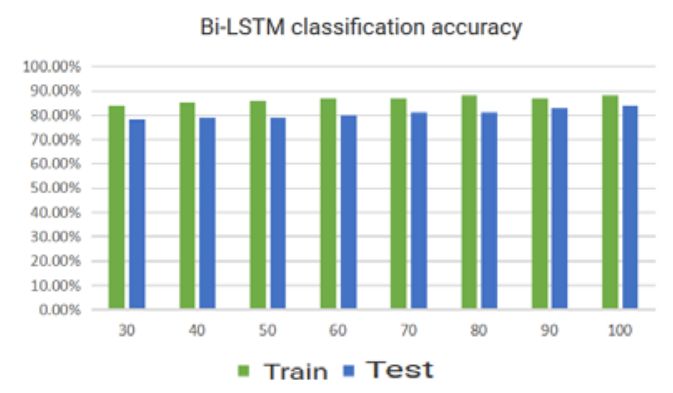

Fig.4 Bi-LSTM classification accuracy

Under the same condition, the data of Bi-LSTM on the input layer is input, the classification accuracy is $80.6 \%$ through the forward propagation and backward propagation of many times, shown as figure 4 . The computing method of Bi-LSTM can speed up the convergence of result and the classification accuracy got is also promoted, compared with the less training time of other model training.

\subsection{The classification accuracy under the different neutral network}

Compared with other neutral network, LSTM can better process the uncorrelation in timing, and provide data reservation for next procession. Bi-LSTM has the advantage from front to back and performs very good in many non casual system. Figure 5 is the comparison of classification accuracy of EEG signal under the different neural network.

Classification accuracy under different neural networks

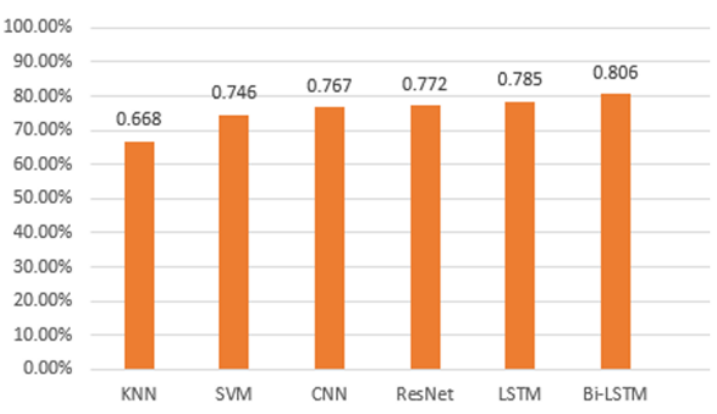

Fig.5 accuracy under different classifications

We can see from the classification consequence, all of them have steady promotion and Bi-LSTM reaches $80.6 \%$ with the highest accuracy due to the advantages of LSTM on sequence signal process, compared with traditional robot study and Convolutional Neural Networks on classification accuracy. 


\section{Conclusion}

This essay puts forward the EEG classification model of depression under the P300 based on Event-related potential to study the classification of P300. This study firstly is starting from EEG induced paradigm from 32 channels to select 16 channel data with more strong robustness and extract the feature of data, and then enter different neutral network models for comparing with classification accuracy. Bi-LSTM model performs relatively excellent and classification accuracy is $80.6 \%$.

\section{Acknowledgement}

This work was carried out by Hebei University of Technology. We gratefully acknowledge the invaluable contribution of Pro.Fu Zhigang and the anonymous reviewers for their careful work and thoughtful suggestions that have helped improve this paper substantially.

\section{References}

1. Yang Yelang, Zhang Qingpin. the study development of depression disease[J]. Public science and technology,020.009(2018):66-68.

2. Xu Wangswang, Lou Zongze, Hou Yanbin, et al. the depression study progress of quantity treatment [J]. Zhejiang Medical Journal,40.18(2018):2080-2082.

3. Picton, T. W., et al. Guidelines for using human eventrelated potentials to study cognition: Recording standards and publication criteria. Psychophysiology 37.2(2000):127-152.

4. Krompinger, Jason W., and R. F. Simons. Cognitive inefficiency in depressive undergraduates: Stroop processing and ERPs. biological psychology 86.3(2011):0-246.

5. Ilan AB, Polich J. P300 and response time from a manual Stroop task. Clin Neurophysiol. 1999,110: 367-373 .

6. Hansenne M,Pitchot W, Gonzalez Moreno A,et al. Suicidal behavior in depressive disorder: an eventrelated potential study[J].Biol Psychiatry, 1996, 40(2): 116-122. 